\title{
Measuring the influence and impact of competitiveness research: a Web of Science approach
}

\begin{abstract}
The purpose of this paper is to measure the influence and impact of competitiveness research by identifying the 100 most cited articles in competitiveness that are published in academic journals indexed in the database of Web of Science of the Institute for Scientific Information between 1980 and 2013. Using citation analysis we investigated the number of citations that were made to the 100 most cited articles that deal with competitiveness during this 34 year period. We also identified articles, authors, journals, institutions, and countries that have had the most contribution to the literature of competitiveness. Further, we determined in which categories of Web of Science these articles were published and how is the time distribution of their publication. Additionally, we investigated the level of competitiveness that has received the most attention, and the latest level of analysis in competitiveness research. We also explored the type of research design these articles used. Finally, we determined the most popular topics covered and the type of firm or industry/name of nation or region analyzed by these articles. The findings of this research provide a reliable basis for competitiveness researchers to better plan their studies and enhance the influence and impact of their research works. However, the most cited articles published in other databases and categories, and citation to these articles in other publications and resources may deserve future research attention.
\end{abstract}

Keyword: Competitiveness; Most cited articles; Citation analysis; Web of Science 
